\title{
The partial constraint satisfaction problem : facets and lifting theorems
}

Citation for published version (APA):

Koster, A. M. C. A., van Hoesel, C. P. M., \& Kolen, A. W. J. (1997). The partial constraint satisfaction problem : facets and lifting theorems. METEOR, Maastricht University School of Business and Economics. METEOR Research Memorandum No. 005 https://doi.org/10.26481/umamet.1997005

Document status and date:

Published: 01/01/1997

DOI:

10.26481/umamet.1997005

Document Version:

Publisher's PDF, also known as Version of record

\section{Please check the document version of this publication:}

- A submitted manuscript is the version of the article upon submission and before peer-review. There can be important differences between the submitted version and the official published version of record.

People interested in the research are advised to contact the author for the final version of the publication, or visit the DOI to the publisher's website.

- The final author version and the galley proof are versions of the publication after peer review.

- The final published version features the final layout of the paper including the volume, issue and page numbers.

Link to publication

\footnotetext{
General rights rights.

- You may freely distribute the URL identifying the publication in the public portal. please follow below link for the End User Agreement:

www.umlib.nl/taverne-license

Take down policy

If you believe that this document breaches copyright please contact us at:

repository@maastrichtuniversity.nl

providing details and we will investigate your claim.
}

Copyright and moral rights for the publications made accessible in the public portal are retained by the authors and/or other copyright owners and it is a condition of accessing publications that users recognise and abide by the legal requirements associated with these

- Users may download and print one copy of any publication from the public portal for the purpose of private study or research.

- You may not further distribute the material or use it for any profit-making activity or commercial gain

If the publication is distributed under the terms of Article $25 \mathrm{fa}$ of the Dutch Copyright Act, indicated by the "Taverne" license above, 


\title{
The Partial Constraint Satisfaction Problem: Facets and Lifting Theorems
}

\author{
Arie M.C.A. Koster ${ }^{1,2}$ \\ Stan P.M. van Hoesel ${ }^{1,3}$ \\ Antoon W.J. Kolen ${ }^{1,4}$
}

January 30,1997

\begin{abstract}
In this paper the partial constraint satisfaction problem (PCSP) is introduced and formulated as a $\{0,1\}$-programming problem. We define the partial constraint satisfaction polytope as the convex hull of feasible solutions for this programming problem. As examples of the class of problems studied we mention the frequency assignment problem and the maximum satisfiability problem. Lifting theorems are presented and some classes of facet-defining valid inequalities for PCSP are given. Computational results show that these valid inequalities reduce the gap between LP-value and IP-value substantially.
\end{abstract}

\section{The partial constraint satisfaction problem}

A partial constraint satisfaction problem is defined by $\left(G=(V, E), D_{V}, P_{E}, Q_{V}\right)$, where $G=$ $(V, E)$ is a connected graph called the constraint graph, $D_{V}$ is a set of domains $D_{v}, v \in V$ where each domain is a finite set, $P_{E}$ is a set of (edge-)penalty functions $P_{\{v, w\}}:\left\{\left\{d_{v}, d_{w}\right\} \mid d_{v} \in\right.$ $\left.D_{v}, d_{w} \in D_{w}\right\} \rightarrow \mathbb{R},\{v, w\} \in E$, and $Q_{V}$ is a set of (vertex-)penalty functions $Q_{v}: D_{v} \rightarrow \mathbb{R}$, $v \in V$.

The partial constraint satisfaction problem is to select exactly one value $d_{v}$ in the domain $D_{v}$ for every $v \in V$ so as to minimize the total sum of the penalties, i.e. $\sum_{\{v, w\} \in E} P_{\{v, w\}}\left(\left\{d_{v}, d_{w}\right\}\right)+$ $\sum_{v \in V} Q_{v}\left(d_{v}\right)$.

The Frequency Assignment Problem (FAP) belongs to the class of partial constraint satisfaction problems. For the FAP a vertex corresponds to a base station, i.e. a directional antenna, in a mobile telephone network. The domain of a vertex is the set of frequencies that can be assigned to that base station, and an edge indicates that communication from one base station defining

\footnotetext{
${ }^{1}$ Dept of Quantitative Economics, Maastricht University, P.O.Box 616, 6200 MD Maastricht, The Netherlands.

2e-mail: A.Koster@KE.UniMaas.NL; home page: http://www.unimaas.nl/ akoster/index.html

${ }^{3}$ e-mail: S.vanHoesel@KE.UniMaas.NL

${ }^{4}$ e-mail: A.Kolen@KE.UniMaas.NL
} 
the edge may interfere with communication from the other base station defining the edge. In most applications interference occurs whenever the distance between the frequencies assigned to the stations is less than a given threshold depending on the two base stations. The penalty of an edge reflects the priority with which interference should be avoided, whereas the penalty on a vertex can be seen as a level of preference for the frequencies.

For another type of frequency assignment problems, involving receiver-transmitter pairs of radio links, that can be formulated as a partial constraint satisfaction problem, we refer to Kolen [2].

The Maximum Satisfiability Problem (MAX SAT) can be formulated as a partial constraint satisfaction problem. In a MAX SAT problem $m$ clauses $c_{1}, \ldots, c_{m}$ involving the boolean variables $x_{1}, \ldots, x_{n}$ are given. Each clause contains a number of literals, where a literal is either a variable or the negation of a variable. The problem is to assign a value true or false to each variable so as to maximize the number of clauses that are satisfied. A clause is satisfied if at least one literal in it has the value true.

It is not straightforward to model MAX SAT as a partial constraint satisfaction problem. We introduce a vertex $v_{c_{i}}$ for every clause $c_{i}, i=1, \ldots, m$ and a vertex $v_{x_{j}}$ for every variable $x_{j}$, $j=1, \ldots, n$. The domain of $v_{c_{i}}$ contains a value for each literal in the clause $c_{i}$, let us denote this value by the literal itself. The domain of $v_{x_{j}}$ is given by $\{$ true, false $\}$. There is an edge between a vertex $v_{c_{i}}$ representing clause $c_{i}$, and a vertex $v_{x_{j}}$ representing variable $x_{j}$ if and only if $x_{j} \in c_{i}$ or $\bar{x}_{j} \in c_{i}\left(\bar{x}_{j}\right.$ is the negation of $\left.x_{j}\right)$. If $x_{j} \in c_{i}$, then the penalty of the combination of domain values $\left(x_{j}\right.$, false $)$ is equal to 1 . If $\bar{x}_{j} \in c_{i}$, then the penalty of the combination of domain values $\left(\bar{x}_{j}\right.$, true $)$ is equal to 1 . All other penalties are zero.

The optimal value of this partial constraint satisfaction problem is $k$ if and only if the optimal value of the corresponding MAX SAT is $m-k$. Furthermore, an optimal solution of the MAX SAT is given by the domain values selected for the vertices corresponding to the variables in the optimal solution of the partial constraint satisfaction problem. This shows that the two problems are equivalent. Since MAX 2 SAT (each clause contains at most 2 literals) is NP-hard (Garey, Johnson and Stockmeyer [1]) a binary constraint satisfaction problem with $\left|D_{v}\right|=2$ for all $v \in V$ is already NP-hard.

For the MAX 2 SAT problem a more compact formulation is possible. We have a vertex $v_{x_{j}}$ corresponding to every variable $x_{j}$ and the domain is given by $\{$ true, false $\}$. There is an edge $\left\{v_{x_{i}}, v_{x_{j}}\right\}$ if and only if there exists a clause containing a literal corresponding to $x_{i}$ and a literal corresponding to $x_{j}$. The penalty corresponding to a combination of values for the variables $x_{i}$ and $x_{j}$ is equal to the number of clauses containing literals corresponding to both variables for which the given combination does not satisfy the clause.

The satisfiability problem (SAT), in which the question is whether there is an assignment of the variables for which all clauses are satisfied, can also be formulated as a partial constraint satisfaction problem as follows. There is one vertex for every clause and an edge if the two corresponding clauses contain a conflicting literal corresponding to the same variable. A combination $\left\{x_{i}, \bar{x}_{i}\right\}$ with $x_{i} \in C_{j}$ and $\bar{x}_{i} \in C_{k}$ has penalty one. All combinations corresponding to nonconflicting literals have penalty zero. The problem is satisfiable if and only if the corresponding 
partial constraint satisfaction problem has optimal value zero.

In Section 2 of this paper we formulate the partial constraint satisfaction problem as $\{0,1\}$ programming problem, we state the dimension of the problem and describe the trivial facet defining valid inequalities. We prove theorems for lifting facets of a subproblem to facets for the original problem in Section 3. In Section 4 we define some classes of facets for the PCSP. Some preliminary computational results are addressed in Section 5, whereas the last section contains the concluding remarks.

\section{Formulation, Dimension and Trivial Facets}

To formulate the partial constraint satisfaction problem as a $\{0,1\}$-programming problem we introduce the following $\{0,1\}$-variables for all $v \in V$ and $d_{v} \in D_{v}$

$$
y\left(v, d_{v}\right) \quad= \begin{cases}1 & \text { if } d_{v} \in D_{v} \text { is selected } \\ 0 & \text { otherwise }\end{cases}
$$

and for all $\{v, w\} \in E, d_{v} \in D_{v}$ and $d_{w} \in D_{w}$

$$
x\left(v, d_{v}, w, d_{w}\right)= \begin{cases}1 & \text { if }\left(d_{v}, d_{w}\right) \in D_{v} \times D_{w} \text { is selected } \\ 0 & \text { otherwise }\end{cases}
$$

Note that since the constraint graph is undirected $x\left(v, d_{v}, w, d_{w}\right)$ and $x\left(w, d_{w}, v, d_{w}\right)$ denote the same variable. To be consistent with the way we denote the $x$ and $y$-variables, let $p\left(v, d_{v}, w, d_{w}\right)$ and $q\left(v, d_{v}\right)$ denote $P_{\{v, w\}}\left(\left\{d_{v}, d_{w}\right\}\right)$ and $Q_{v}\left(d_{v}\right)$, respectively.

A $\{0,1\}$-programming formulation of the binary constraint satisfaction problem is given by

$$
\begin{array}{lll}
\min & \sum_{\{v, w\} \in E} \sum_{d_{v} \in D_{v}} \sum_{d_{w} \in D_{w}} p\left(v, d_{v}, w, d_{w}\right) x\left(v, d_{v}, w, d_{w}\right) \\
& +\sum_{v \in V} \sum_{d_{v} \in D_{v}} q\left(v, d_{v}\right) y\left(v, d_{v}\right) & \\
\text { s.t. } & \sum_{d_{v} \in D_{v}} y\left(v, d_{v}\right)=1 & \forall v \in V \\
& \sum_{d_{w} \in D_{w}} x\left(v, d_{v}, w, d_{w}\right)=y\left(v, d_{v}\right) & \forall\{v, w\} \in E, d_{v} \in D_{v} \\
& x\left(v, d_{v}, w, d_{w}\right) \in\{0,1\} & \forall\{v, w\} \in E, d_{v} \in D_{v}, d_{w} \in D_{w} \\
& y\left(v, d_{v}\right) \in\{0,1\} & \forall v \in V, d_{v} \in D_{v}
\end{array}
$$

Constraints (2) model the fact that exactly one value in the domain of a vertex should be selected. Constraints (3) model the fact that the combination of values selected for an edge should be consistent with the values selected for the vertices of that edge. 
We define the partial constraint satisfaction polytope $X(P C S P)$ to be the convex hull of all $\{0,1\}$-vectors $(y, x)$ satisfying (2) and (3). Although the $y$-variables can be eliminated from the formulation, we have found it more convenient to keep them in the formulation. Note that once the $y$-variables are $\{0,1\}$ the $x$-variables are forced to be $\{0,1\}$. Therefore, the $x$-variables can be relaxed to be $[0,1]$-variables.

The dimension of the binary constraint satisfaction polytope is given by Theorem 2.1.

Theorem 2.1 The dimension of $X(P C S P)$, defined by $\left(G=(V, E), D_{V}\right)$ is

$$
\sum_{v \in V}\left(\left|D_{v}\right|-1\right)+\sum_{\{v, w\} \in E}\left(\left|D_{v}\right|-1\right)\left(\left|D_{w}\right|-1\right)
$$

Proof. We will first prove that the dimension is less than or equal to the given value by defining

$$
\begin{gathered}
\sum_{v \in V}\left|D_{v}\right|+\sum_{\{v, w\} \in E}\left|D_{v}\right|\left|D_{w}\right|(=\text { number of variables }) \\
-\quad\left(\sum_{v \in V}\left(\left|D_{v}\right|-1\right)+\sum_{\{v, w\} \in E}\left(\left|D_{v}\right|-1\right)\left(\left|D_{w}\right|-1\right)\right. \\
=|V|+\sum_{\{v, w\} \in E}\left(\left|D_{v}\right|+\left|D_{w}\right|-1\right)
\end{gathered}
$$

linearly independent equalities which are satisfied by all solutions of PCSP. These linear independent equalities are obtained by taking all constraints (2), and for every edge $\{v, w\}$ all but one of the constraints (3). The constraints (3) for a given edge $\{v, w\}$ can be viewed as the constraints of a transportation problem with suppliers indicated by $\left(v, d_{v}\right)$ with supply $y\left(v, d_{v}\right)$ and clients indicated by $\left(w, d_{w}\right)$ with demand $y\left(w, d_{w}\right)$. It is well-known that deleting one of these constraints results in a set of linear independent equalities.

Next, we will prove that the dimension is greater than or equal to the given value by defining $1+\sum_{v \in V}\left(\left|D_{v}\right|-1\right)+\sum_{\{v, w\} \in E}\left(\left|D_{v}\right|-1\right)\left(\left|D_{w}\right|-1\right)$ affinely independent solutions. Note that once the $y$-variables are given, the $x$-variables are uniquely determined by constraints (3). To define these solutions we arbitrarily select a value $d_{v}^{*} \in D_{v}$. One solution is given by $y\left(v, d_{v}^{*}\right)=1$ for all $v \in V$.

For each $v \in V$ and $d_{v} \in D_{v} \backslash\left\{d_{v}^{*}\right\}$, we define the solution $y\left(v, d_{v}\right)=1, y\left(w, d_{w}^{*}\right)=1$ for all $w \neq v$. Note that there are $\sum_{v \in V}\left(\left|D_{v}\right|-1\right)$ solutions of this type.

For each $\{v, w\} \in E, d_{v} \in D_{v} \backslash\left\{d_{v}^{*}\right\}$, and $d_{w} \in D_{w} \backslash\left\{d_{w}^{*}\right\}$, we define the solution $y\left(v, d_{v}\right)=$ $y\left(w, d_{w}\right)=1$ and $y\left(u, d_{u}^{*}\right)=1$ for all $u \in V, u \neq v, u \neq w$.

Note that there are $\sum_{\{v, w\} \in E}\left(\left|D_{v}\right|-1\right)\left(\left|D_{w}\right|-1\right)$ solutions of this type. These solutions are affinely independent because the $\sum_{v \in V}\left(\left|D_{v}\right|-1\right)+\sum_{\{v, w\} \in E}\left(\left|D_{v}\right|-1\right)\left(\left|D_{w}\right|-1\right)$ vectors obtained by subtracting the first solution from all other solutions are linearly independent. To see this note that each vector has a one in a component in which each previously defined vector has a zero. For the solution defined by $\left(v, d_{v}\right)$ take the component corresponding to $x\left(v, d_{v}, w, d_{w}^{*}\right)$ or $x\left(w, d_{w}^{*}, v, d_{v}\right)$ for an edge $\{w, v\} \in E$. For the solution defined by $\{v, w\} \in E, d_{v} \in D_{v}$, $d_{w} \in D_{w}$ take the component corresponding to $x\left(v, d_{v}, w, d_{w}\right)$. 
It follows straightforward that the non-negativity constraints of the $x$-variables define facets of the polytope if both domains have at least two elements.

Theorem 2.2 For every $\{v, w\} \in E,\left|D_{v}\right| \geq 2,\left|D_{w}\right| \geq 2, d_{v} \in D_{v}, d_{w} \in D_{w}$ the inequality

$$
x\left(v, d_{v}, w, d_{w}\right) \geq 0
$$

defines a facet for $X(P C S P)$.

Proof. In the proof of Theorem 2.1 we listed $\operatorname{dim} X(P C S P)+1$ affinely independent solutions exactly one of which has $x\left(v, d_{v}, w, d_{w}\right)=1\left(d_{v} \neq d_{v}^{*}, d_{w} \neq d_{w}^{*}\right)$ and all others have $x\left(v, d_{v}, w, d_{w}\right)=0$. Hence, we have $\operatorname{dim} X(P C S P)$ affinely independent solutions satisfying $x\left(v, d_{v}, w, d_{w}\right) \geq 0$ with equality.

\section{$3 \quad$ Lifting theorems}

In this section we will discuss two different types of lifting. Firstly, we show that a facet defining inequality of a partial constraint satisfaction problem defined by the constraint graph $G=(V, E)$ and a set of domains $D_{v}, v \in V$ also defines a facet for the partial constraint satisfaction problem defined by any constraint graph for which $G=(V, E)$ is an induced subgraph, and a set of domains where the domain for a vertex $v \in V$ is unchanged and all other vertices have a domain of cardinality one. If $X(P C S P)$ is defined by $\left(G=(V, E), D_{V}\right)$, let $X_{u}(P C S P)$ denote the PCSP-polytope defined by the induced subgraph on $V \backslash\{u\}$ with the same domains.

Theorem 3.1 Let $X(P C S P)$ be defined by $\left(G=(V, E), D_{V}\right)$ with $\left|D_{u}\right|=1$, for some $u \in V$. If $\pi x \leq \pi_{0}$ is a facet defining inequality for $X_{u}(P C S P)$, then $\pi x \leq \pi_{0}$ is a facet defining inequality for $X(P C S P)$.

Proof. The polytopes belonging to both problems have the same dimension.

Next, we show how a facet defining inequality of a constraint satisfaction problem defined by the constraint graph $G=(V, E)$ and a set of domains $D_{v}, v \in V$ can be lifted into a facet defining inequality for the constraint satisfaction problem by the same constraint graph and set of domains $D_{v}^{\prime}, v \in V$, where $D_{v}^{\prime}=D_{v}$, for all $v \in V, v \neq u$, and $D_{u}^{\prime}=D_{u} \cup\left\{d_{u}^{\prime}\right\}$.

Theorem 3.2 states that if we make $d_{u}^{\prime}$ a copy of any domain element $d_{u} \in D_{u}$ (i.e. the coefficient of $x\left(u, d_{u}^{\prime}, v, d_{v}\right)$ is equal to the coefficient of $x\left(u, d_{u}, v, d_{v}\right)$, for all $v \in \delta_{u}, d_{v} \in D_{v}$, where $\delta_{u}$ defines the set of neighbours of $u$ in the constraint graph; $\delta_{u}=\{v \mid\{u, v\} \in E\}$ ), then the new inequality is facet defining for the extended problem whenever the original inequality is facet defining for the original problem. 
In order to prove Theorem 3.2 we need Lemma 3.1 and Lemma 3.2. The components corresponding to $\left(v, d_{v}\right)$ are given by $x\left(v, d_{v}, w, d_{w}\right)$ for all $w \in \delta_{v}, d_{w} \in D_{w}$ and $y\left(v, d_{v}\right)$.

Lemma 3.1 If $\sum_{v \in \delta_{u}} \sum_{d_{v} \in D_{v}} \alpha\left(u, d_{u}, v, d_{v}\right) x\left(u, d_{u}, v, d_{v}\right) \geq 0, u \in V, d_{u} \in D_{u}$, is a facet defining valid inequality for $X(P C S P)$, then the inequality describes a trivial facet.

Proof. We first prove that by adding implicit equalities of the PCSP the valid inequality can be rewritten as $\sum_{v \in \delta_{u}} \sum_{d_{v} \in D_{v}} \beta\left(u, d_{u}, v, d_{v}\right) x\left(u, d_{u}, v, d_{v}\right) \geq 0$ with $\beta\left(u, d_{u}, v, d_{v}\right) \geq 0$.

Let $d_{v}^{*} \in D_{v}, v \in \delta_{u}$, be such that $\alpha\left(u, d_{u}, v, d_{v}^{*}\right)=\min _{d_{v} \in D_{v}}\left\{\alpha\left(u, d_{u}, v, d_{v}\right)\right\}$. We add the implicit equalities $\alpha\left(u, d_{u}, v, d_{v}^{*}\right)\left(y\left(u, d_{u}\right)-\sum_{v \in D_{v}} x\left(u, d_{u}, v, d_{v}\right)\right)=0$ to the inequality. We obtain a valid inequality of the form

$$
\begin{aligned}
& \sum_{v \in \delta_{u}} \alpha\left(u, d_{u}, v, d_{v}^{*}\right) y\left(u, d_{u}\right)+ \\
& \sum_{v \in \delta_{u}} \sum_{d_{v} \in D_{v}}\left(\alpha\left(u, d_{u}, v, d_{v}\right)-\alpha\left(u, d_{u}, v, d_{v}^{*}\right)\right) x\left(u, d_{u}, v, d_{v}\right) \geq 0
\end{aligned}
$$

From the solution in which we select $d_{v}^{*} \in D_{v}$ for all $v \in \delta_{u}$ and $d_{u} \in D_{u}$ for $u$, and the validity of $\alpha x \geq 0$ it follows that $\sum_{v \in \delta_{u}} \alpha\left(u, d_{u}, v, d_{v}^{*}\right) \geq 0$. Substitute $y\left(u, d_{u}\right)=\sum_{d_{v} \in D_{v}} x\left(u, d_{u}, v, d_{v}\right)$ for some $v \in \delta_{u}$ and we obtain an inequality where each coefficient is nonnegative.

Since $x\left(u, d_{u}, v, d_{v}\right) \geq 0$ is valid for all $\{u, v\} \in E, d_{u} \in D_{u}, d_{v} \in D_{v}$ it follows that the dimension of the face of the inequality is maximal if there is exactly one nonzero coefficient $\beta\left(u, d_{u}, v, d_{v}\right)$. In that case the inequality defines the same face as the inequality $x\left(u, d_{u}, v, d_{v}\right) \geq 0$.

Lemma 3.2 Let $\pi x \leq \pi_{0}$ define a non-trivial facet of $X(P C S P)$. Then for each $u \in V$, $d_{u} \in D_{u}$ there are exactly $q=1+\sum_{v \in \delta_{u}}\left(\left|D_{u}\right|-1\right)$ solutions with $y\left(u, d_{u}\right)=1$ and $\pi x=\pi_{0}$ which are affinely independent with respect to the components corresponding to $\left(u, d_{u}\right)$.

Proof. Let $\left(y^{1}, x^{1}\right), \ldots,\left(y^{p}, x^{p}\right)$ be $p=\operatorname{dim} X(P C S P)$ affinely independent solutions which satisfy $\pi x \leq \pi_{0}$ with equality. Moreover, let $\left(y^{1}, x^{1}\right), \ldots,\left(y^{q}, x^{q}\right)$ be the solutions with $y\left(u, d_{u}\right)=$ 1 which are affinely independent with respect to the components $y\left(u, d_{u}\right)$ and $x\left(u, d_{u}, v, d_{v}\right)$ for all $v \in \delta_{u}, d_{v} \in D_{v}$. Note that by $y\left(u, d_{u}\right)=1$ these solutions are also linearly independent. We prove that the corresponding matrix $A$ with $1+\sum_{v \in \delta_{u}}\left|D_{v}\right|$ rows and $q$ columns has rank $1+\sum_{v \in \delta_{u}}\left(\left|D_{v}\right|-1\right)$, which implies that there are exactly $1+\sum_{v \in \delta_{u}}\left(\left|D_{v}\right|-1\right)$ solutions which are affinely independent with respect to these components.

To prove that the matrix has rank $1+\sum_{v \in \delta_{u}}\left(\left|D_{v}\right|-1\right)$ we will prove that there are exactly $\left|\delta_{u}\right|$ linear independent vectors $\alpha$ such that $\alpha A=0$. Every column of $A$ satisfies $y\left(u, d_{u}\right)=$ $\sum_{d_{v} \in D_{v}} x\left(u, d_{u}, v, d_{u}\right)$ for all $v \in \delta_{u}$. Therefore there are at least $\left|\delta_{u}\right|$ linear independent vectors $\alpha$ such that $\alpha A=0$. Assume there exists another vector $\alpha$ such that $\alpha A=0$ which is linear independent from the other $\left|\delta_{u}\right|$ linear independent vectors. If the coefficient of $y\left(u, d_{u}\right)$ is nonzero, then we use one of the equalities $y\left(u, d_{u}\right)=\sum_{d_{v} \in D_{v}} x\left(u, d_{u}, v, d_{v}\right)$ to eliminate this coefficient. 
Since all solutions $\left(y^{1}, x^{1}\right),\left(y^{2}, x^{2}\right), \ldots,\left(y^{p}, x^{p}\right)$ satisfy $\alpha x=0$ it follows that $\{x \in X \mid \pi x=$ $\left.\pi_{0}\right\} \subseteq\{x \in X \mid \alpha x=0\}$. If equality does not hold, then $\{x \in X \mid \alpha x=0\}=X$ and $\alpha x=0$ is an implicit equality. However, $\alpha$ is linear independent from the implicit equalities involving $x\left(u, d_{u}, v, d_{u}\right), v \in \delta_{u}, d_{v} \in D_{v}$. Hence $\{x \in X \mid \alpha x=0\}=\left\{x \in X \mid \pi x=\pi_{0}\right\}$. It follows that either $\alpha x \geq 0$ or $\alpha x \leq 0$ is a valid inequality for $X(P C S P)$ defining the same facet as $\pi x \leq \pi_{0}$. Without loss of generality (multiply $\alpha$ by -1 if necessary) assume $\alpha x \geq 0$ for all $x \in X$. It is proved in Lemma 3.1 that in that case $\pi x \leq \pi_{0}$ defines a trivial facet.

Now, we can prove the main theorem of this paper.

Theorem 3.2 Let $X(P C S P)$ be defined by $\left(G=(V, E), D_{V}\right)$. Let $u \in V, d_{u} \in D_{u}$. Define $X^{\prime}(P C S P)$ by $\left(G(V, E), D_{V}^{\prime}\right)$ with $D_{v}^{\prime}=D_{v}, v \in V \backslash\{u\}, D_{u}^{\prime}=D_{u} \cup\left\{d_{u}^{\prime}\right\}$. If $\pi x \leq \pi_{0}$ is a non-trivial facet defining inequality for $X(P C S P)$, then

$$
\pi x+\sum_{v \in \delta(u)} \sum_{d_{v} \in D_{v}} \pi\left(u, d_{u}, v, d_{v}\right) x\left(u, d_{u}^{\prime}, v, d_{v}\right) \leq \pi_{0}
$$

is facet defining for $X^{\prime}(P C S P)$.

Proof. First, note that $\operatorname{dim} X^{\prime}(P C S P)=\operatorname{dim} X(P C S P)+1+\sum_{v \in \delta_{u}}\left(\left|D_{v}\right|-1\right)$. Let the solutions $\left(y^{1}, x^{1}\right), \ldots,\left(y^{p}, x^{p}\right)$, where $p=\operatorname{dim} X(P C S P)$, be a set of affinely independent solutions which satisfy $\pi x \leq \pi_{0}$ with equality. It follows from Lemma 3.2 that there exist $1+\sum_{v \in \delta_{u}}\left(\left|D_{v}\right|-1\right)$ solutions which satisfy $y\left(u, d_{u}\right)=1$ and are affinely independent with respect to $\left(u, d_{u}\right)$. Replace in these solutions $d_{u}$ by $d_{u}^{\prime}$. Then these new solutions together with the old solutions are affinely independent.

In Section 4 we will define some facet defining inequalities for a partial constraint satisfaction problem defined by $G=(V, E)$ and a set of domains $D_{v}, v \in V$. To prove that they are facet defining we will first prove that they are facet defining for a constraint satisfaction problem defined by an induced subgraph $G_{S}=\left(S, E_{S}\right)$ of $G$ and a set of domains $D_{v}$, with $\left|D_{v}\right|=2$, $v \in S$. Next, Theorems 3.1 and 3.2 are used to extend these facets defining inequalities to facet defining inequalities for the original problem.

\section{Non-trivial facets of the PCSP}

The non-trivial facets we will describe in this section are characterized by an induced subgraph $G_{S}=\left(S, E_{S}\right)$ of the constraint graph $G$. For every $v \in S$ the domain $D_{v}$ is partitioned into $A_{v}$ and $B_{v}$. Domain values in $A_{v}$ are copies of one another; likewise the domain values in $B_{v}$. Therefore to describe the facets it is sufficient to specify for each edge $\{v, w\} \in E_{S}$ the coefficients $a a(v, w), a b(v, w), b a(v, w)$ and $b b(v, w)$ corresponding to the coefficient of $x\left(v, d_{v}, w, d_{w}\right)$ with respectively $\left\{d_{v}, d_{w}\right\} \in A_{v} \times A_{w},\left\{d_{v}, d_{w}\right\} \in A_{v} \times B_{w},\left\{d_{v}, d_{w}\right\} \in B_{v} \times A_{w}$ and $\left\{d_{v}, d_{w}\right\} \in B_{v} \times B_{w}$. 
The facet takes the form

$$
\begin{aligned}
\sum_{\{v, w\} \in E_{S}}( & \sum_{d_{v} \in A_{v}} \sum_{d_{w} \in A_{w}} a a(v, w) x\left(v, d_{v}, w, d_{w}\right)+ \\
& \sum_{d_{v} \in A_{v}} \sum_{d_{w} \in B_{w}} a b(v, w) x\left(v, d_{v}, w, d_{w}\right)+ \\
& \sum_{d_{v} \in B_{v}} \sum_{d_{w} \in A_{w}} b a(v, w) x\left(v, d_{v}, w, d_{w}\right)+ \\
& \left.\sum_{d_{v} \in B_{v}} \sum_{d_{w} \in B_{w}} b b(v, w) x\left(v, d_{v}, w, d_{w}\right)\right) \quad \sigma \quad c
\end{aligned}
$$

where $\sigma \in\{\geq, \leq\}$.

It follows from the lifting theorems in Section 3 that in order to prove that an inequality of type (8) is facet defining it is sufficient to prove that (8) is facet defining for $X(P C S P)$ defined by $G_{S}=\left(S, E_{S}\right)$ and $A_{v}=\left\{a_{v}\right\}, B_{v}=\left\{b_{v}\right\}$, for all $v \in S$.

\subsection{The cycle-inequality}

Firstly, we introduce the cycle-inequality. A $k$-cycle inequality, $k \geq 3$, is defined by

$$
\begin{aligned}
& S \quad=\left\{v_{i} \mid i=1, \ldots, k\right\} \\
& \left.E_{S}=\left\{\left\{v_{i}, v_{i+1}\right\} \mid i=1, \ldots, k-1\right\}\right\} \cup\left\{\left\{v_{k}, v_{1}\right\}\right\} \\
& a a\left(v_{i}, v_{i+1}\right)=b b\left(v_{i}, v_{i+1}\right)=1, a b\left(v_{i}, v_{i+1}\right)=b a\left(v_{i}, v_{i+1}\right)=0 \\
& a a\left(v_{k}, v_{1}\right)=b b\left(v_{k}, v_{1}\right)=0, a b\left(v_{k}, v_{1}\right)=b a\left(v_{k}, v_{1}\right)=1 \\
& \begin{array}{l}
\sigma \quad=\leq \\
c \quad=k-1
\end{array}
\end{aligned}
$$

We will call a domain value $d_{v} \in D_{v}$ an a-value whenever $d_{v} \in A_{v}$; otherwise it is a $b$-value. Figure 1 shows the 3-cycle and 4-cycle inequality. The a-dot represents the $A$-subset of the domain; the $b$-dot represents the $B$-subset of the domain. A line between two dots indicate that the coefficient corresponding to the indicated two subsets is equal to one.

Theorem 4.1 The $k$-cycle inequality, $k \geq 3$, is valid for $X(P C S P)$.

Proof. Consider a solution. Each edge of the cycle contributes at most one to the left hand side of (8). If $d_{v_{k}}$ and $d_{v_{1}}$ are a-values, then the edge $\left\{v_{1}, v_{k}\right\}$ does not contribute to the left hand side of (8) and hence (8) is satisfied. The same can be applied if $d_{v_{k}}$ and $d_{v_{1}}$ are b-values.

If $d_{v_{1}}$ is an a-value and $d_{v_{k}}$ is a b-value, then there exists an $i, 1 \leq i \leq k-1$, such that $d_{v_{i}}$ is an a-value and $d_{v_{i+1}}$ is a b-value. Hence the edge $\left\{v_{i}, v_{i+1}\right\}$ does not contribute to the left hand side of (8), and hence (8) is satisfied.

If $d_{v_{1}}$ is a b-value and $d_{v_{k}}$ is an a-value, then the same reasoning applies. 


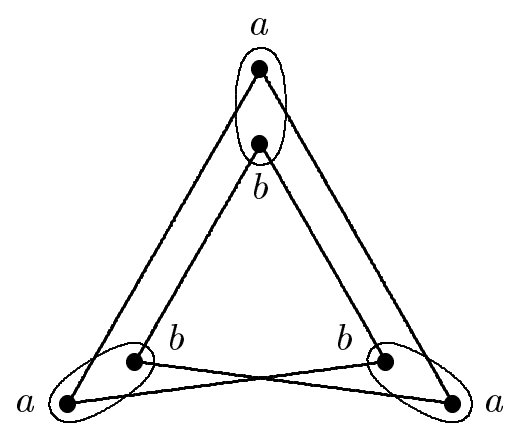

$\leq 2$

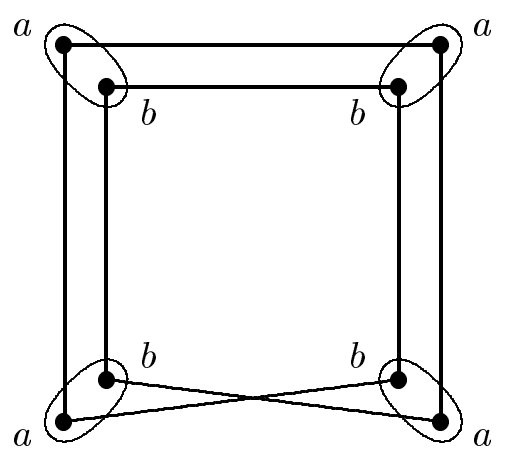

$\leq 3$

Figure 1: Cycle Inequalities

The proof of Theorem 4.1 also indicates the structure of the solutions which satisfy (8) with equality. If $d_{v_{1}}$ and $d_{v_{k}}$ are both a-values, then all other domain values in the cycle must be a-values as well.

If $d_{v_{1}}$ and $d_{v_{k}}$ are both b-values, then all other domain values in the cycle must be b-values as well.

If $d_{v_{1}}$ is an a-value and $d_{v_{k}}$ a b-value, then there exists an $i, 1 \leq i \leq k-1$, such that $d_{v_{j}}$, $1 \leq j \leq i$, is an a-value, and $d_{v_{j}}, i+1 \leq j \leq k$ is a b-value.

If $d_{v_{1}}$ is a b-value and $d_{v_{k}}$ is an a-value, then there exists an $i, 1 \leq i \leq k-1$, such that $d_{v_{j}}, 1 \leq j \leq i$ is a b-value, and $d_{v_{j}}, i+1 \leq j \leq k$ is an a-value.

Theorem 4.2 The $k$-cycle inequality, $k \geq 3$, is facet defining for $X(P C S P)$.

Proof. By the results of Section 3 it is sufficient to prove that the $k$-cycle inequality is facet defining for $X(P C S P)$ defined by the $k$-cycle constraint graph and $A_{v_{i}}=\left\{a_{i}\right\}, B_{v_{i}}=\left\{b_{i}\right\}$, $i=1, \ldots, k$. The dimension of $X(P C S P)$ is $2 k$. The $2 k$ affinely independent solutions satisfying the $k$-cycle inequality with equality are given below. After each solution we have indicated a component for which this solution is the unique solution having a one in this component. This proves that these solutions are affinely independent.

$$
\begin{array}{lll}
\left(a_{1}, \ldots, a_{k}\right) & & \left(x\left(v_{1}, a_{1}, v_{k}, a_{k}\right)\right) \\
\left(a_{1}, \ldots, a_{i}, b_{i+1}, \ldots, b_{k}\right) & i=1, \ldots, k-1 & \left(x\left(v_{i}, a_{i}, v_{i+1}, b_{i+1}\right)\right) \\
\left(b_{1}, \ldots, b_{k}\right) & & \left(x\left(v_{1}, b_{1}, v_{k}, b_{k}\right)\right) \\
\left(b_{1}, \ldots, b_{i}, a_{i+1}, \ldots, a_{k}\right) & i=1, \ldots, k-1 & \left(x\left(v_{i}, b_{i}, v_{i+1}, a_{i+1}\right)\right)
\end{array}
$$




\subsection{The clique-cycle inequality}

A second class of facet defining valid inequalities are the clique-cycle inequalities. A $k$-cliquecycle inequality, $k \geq 3$, is defined by

$$
\begin{array}{lll}
S=\left\{v_{i} \mid i=1, \ldots, k\right\} & \\
E_{S}=\left\{\left\{v_{i}, v_{j}\right\} \mid i, j=1, \ldots, k, i<j\right\} & \\
E_{C}=\left\{\left\{v_{i}, v_{i+1}\right\} \mid i=1, \ldots, k-1\right\} \cup\left\{\left\{v_{k}, v_{1}\right\}\right\} & \text { subset forming a } k \text {-cycle } \\
a a(v, w)=a b(v, w)=b a(v, w)=0, b b(v, w)=1 & \{v, w\} \in E_{S} \backslash E_{C} \\
a a\left(v_{i}, v_{i+1}\right)=a b\left(v_{i}, v_{i+1}\right)=b a\left(v_{i}, v_{i+1}\right)=1, b b\left(v_{i}, v_{i+1}\right)=0 & i=1, \ldots, k-1 \\
a a\left(v_{k}, v_{1}\right)=a b\left(v_{k}, v_{1}\right)=b a\left(v_{k}, v_{1}\right)=1, b b\left(v_{k}, v_{1}\right)=0 & \\
\sigma \quad=\geq & \\
c \quad=k-1 &
\end{array}
$$

Figure 2 shows a clique-cycle inequality for $k=3$ and $k=4$. For $k=3$ the facet described by a clique-cycle inequality is the same as a facet described by a cycle inequality.
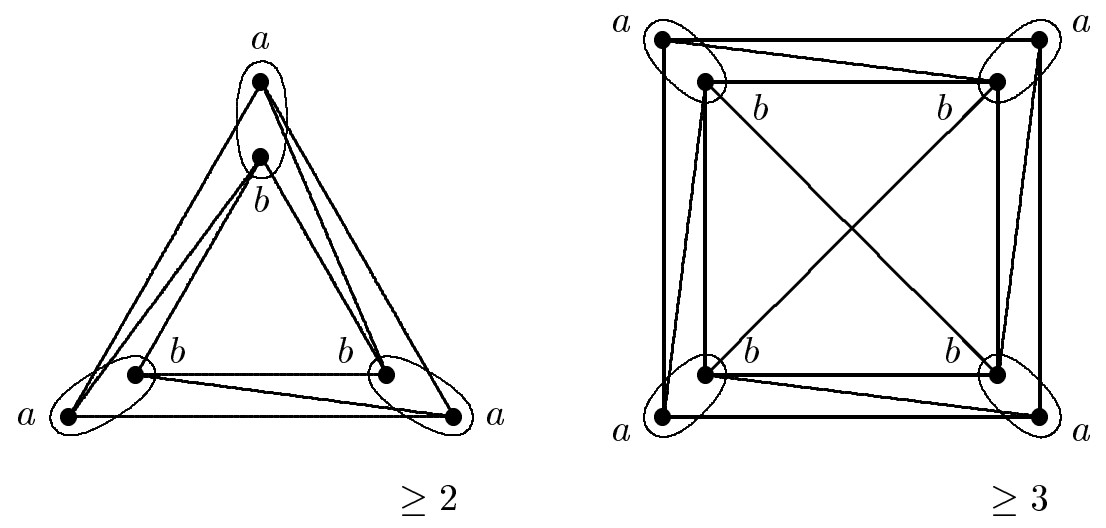

Figure 2: Clique-Cycle Inequality

Theorem 4.3 The $k$-clique-cycle inequality, $k \geq 3$, is valid for $X(P C S P)$.

Proof. Consider an arbitrary solution. Whenever an a-node is selected in $D_{v_{i}}$, then the edge in the $k$-cycle $\left\{v_{i}, v_{i+1}\right\}$ (or $\left\{v_{k}, v_{1}\right\}$ whenever $i=k$ ) contributes exactly one to the left hand side of (8). Hence (8) is valid whenever at least $k-1$ a-nodes are selected. Therefore, let us assume that $k-p$ a-nodes and hence $p$ b-nodes are selected, $p \geq 2$.

If $v$ van $w$ are both b-nodes, then the edge $\{v, w\}$ contributes one to the left hand side of (8). The total contribution of all edges between b-nodes is $p(p-1) / 2$. 
The total contribution of the a-nodes is equal to the number of a-nodes $k-p$. To prove that the total contribution is at least $k-1$ we have to prove that $p(p-1) / 2+(k-p) \geq k-1$, i.e. $p^{2}-3 p+2 \geq 0$ or $(p-1)(p-2) \geq 0$. This holds since $p \geq 2$.

The proof of Theorem 4.3 also indicates the structure of the solutions which satisfy (8) with equality. A solution satisfies (8) with equality if and only if the number of b-nodes selected is either one or two.

Theorem 4.4 The $k$-clique-cycle inequality, $k \geq 3$, is facet defining for $X(P C S P)$.

Proof. By the results of Section 3 it is sufficient to prove that the $k$-clique-cycle inequality is facet defining for $X(P C S P)$ defined by the $k$-clique constraint graph and $A_{v_{i}}=\left\{a_{i}\right\}, B_{v_{i}}=\left\{b_{i}\right\}$, $i=1, \ldots, k$. The dimension of $X(P C S P)$ is $k+k(k-1) / 2$. The $k+k(k-1) / 2$ affinely independent solutions satisfying (8) with equality are given below. For each solution we will also specify a component for which this solution has a value one and all previously defined solutions have value zero at this component. This proves that the solutions are affinely independent.

For each $p=1, \ldots, k$ we define

$v_{p} \quad$ is a b-node

$v_{i} \quad i \neq p$ is an a-node

with component $x\left(v_{p}, b_{p}, v_{p+1}, a_{p+1}\right)\left(x\left(v_{k}, b_{k}, v_{1}, a_{1}\right)\right.$ if $\left.p=k\right)$.

For each $p, q=1, \ldots, k, p<q$, we define

$v_{p} \quad$ is a b-node

$v_{q}$ is a b-node

$v_{i} \quad i \neq p, q$ is an a-node

with component $x\left(v_{p}, b_{p}, v_{q}, b_{q}\right)$

\section{Computational Results}

A first test of the quality of the valid inequalities described above is done on 11 instances with $\left|D_{v}\right|=2$ for $v \in V$. These instances are subproblems of the Frequency Assignment CALMAinstance celar8, which have to be solved in the crossover of the genetic algorithm described by Kolen [2]. We used the callable library of CPLEX 4.0 to solve the linear programming relaxation $\left(z_{L P}\right)$, the $(0,1)$-programming problem $\left(z_{I P}\right)$ as well as the linear programming relaxation with 3cycle valid inequalities $\left(z_{3}\right)$. The selection of violated valid inequalities was done by enumaration of all valid inequalities with $k=3$ (i.e. 4 valid inequalities for each 3 -cycle were available). For 
all instances we have $|V|=458$ and $|E|=1655$. The results are presented in Table 1 . The program written in $\mathrm{C}++$ was running on a DEC 2100 A500MP workstation with $128 \mathrm{Mb}$ internal memory. The table shows that for all instances the LP-relaxation with 3-cycle valid inequalities gives an integer solution. The number of violated inequalities which had to be added is given in the last column. The computation times were in average reduced by $76.4 \%$.

\begin{tabular}{|l||r|r|r||r|r|r|r||r|}
\hline instance & $z_{L P}$ & $z_{3}$ & $z_{I P}$ & $\mathrm{CPU} z_{L P}$ & $\mathrm{CPU} z_{3}$ & $\mathrm{CPU} z_{3+I P}$ & $\mathrm{CPU} z_{I P}$ & \#v.i. \\
\hline \hline c8_1 & 848.5 & 986 & 986 & 8.8 & 18.1 & 18.1 & 78.0 & 1104 \\
c8_2 & 721 & 836 & 836 & 8.7 & 11.4 & 11.4 & 48.4 & 497 \\
c8_3 & 630.5 & 747 & 747 & 7.8 & 13.1 & 13.1 & 63.1 & 771 \\
c8_4 & 802 & 834 & 834 & 8.0 & 10.9 & 10.9 & 35.4 & 1243 \\
c8_5 & 627.5 & 729 & 729 & 7.5 & 11.3 & 11.3 & 35.7 & 608 \\
c8_6 & 695 & 717 & 717 & 8.6 & 12.0 & 12.0 & 31.5 & 907 \\
c8_7 & 836 & 894 & 894 & 8.2 & 9.9 & 9.9 & 39.1 & 267 \\
c8_8 & 757 & 835 & 835 & 7.2 & 10.5 & 10.5 & 71.2 & 747 \\
c8_9 & 769 & 866 & 866 & 9.2 & 12.6 & 12.6 & 54.9 & 610 \\
c8_10 & 768.5 & 812 & 812 & 8.1 & 10.0 & 10.0 & 37.7 & 215 \\
c8_11 & 622 & 814 & 814 & 7.3 & 16.0 & 16.0 & 187.1 & 1259 \\
\hline p1 & 35.5 & 104.5 & 110 & 6.6 & 25.5 & 152.4 & - & 266 \\
\hline
\end{tabular}

Table 1: Computational results

An instance with a large gap between LP and IP was given by a subproblem of a Frequency Assignment Problem of a large telecommunication company. This instance has 708 vertices and 1677 edges (again all domains contains 2 values). The 3 -cycle inequalities close the gap between LP and IP with 92.6\%. With these valid inequalities CPLEX needed 113 nodes branch-andbound nodes to obtain and prove the optimal value. CPLEX was not able to solve this instance to optimality without adding valid inequalities.

\section{Concluding Remarks}

In the case $\left|D_{v}\right|=2$ for all $v \in V$ the number of $k$-cycle inequalities which describe different facets of the polytope is $2^{k-1}$, which give us the possibility the enumerate all valid inequalities for small $k$ (which is done for the instances mentioned in the previous section. However, if the number of domain elements grows the number of available cycle and clique-cycle inequalities which define different facets increases enormously. Therefore, in a future paper the separation problems for each class of valid inequalities will be discussed. Heuristics for these separation problems have to be developed, and have to be implemented in a Branch-and-Cut framework to solve large-size real-life problems (like the CALMA-instances). Moreover, due to the size of these instances, problems will arise in solving the LP relaxation. 


\section{References}

[1] M. Garey, D. Johnson, and L. Stockmeyer. "Some simplified NP-complete graph problems". Theoretical Computer Science, 1:237-267, 1976.

[2] A.W.J. Kolen. "A genetic algorithm for frequency assignment problems". Technical report, Maastricht University, 1997. 